\title{
Efeito da somatotrofina bovina (bST-r), do implante de progestágeno e do desmame por 72 horas na indução do estro e na taxa de prenhez em vacas
}

\author{
[Effect of bovine somatotropin (bST-r), progestagen intravaginal device and temporary calf removal on \\ estrous induction and on pregnancy rate in cows] \\ M.N. Maciel' ${ }^{1}$, J.P. Neves ${ }^{2 *}$, P.B.D. Gonçalves ${ }^{2}$, J.F.C. Oliveira ${ }^{2}$, A.M. Farias ${ }^{3}$ \\ ${ }^{1}$ Doutorando em Medicina Veterinária da Universidade Federal de Santa Maria, Bolsista da CAPES \\ ${ }^{2}$ Departamento de Clínica de Grandes Animais, BioRep \\ Universidade Federal de Santa Maria \\ 97105-900 - Santa Maria, RS \\ ${ }^{3}$ Aluno de Graduação do Curso de Medicina Veterinária, Bolsista de Iniciação Científica, UFSM
}

Recebido para publicação em 7 de novembro de 2000.

Recebido para publicação, após modificações, em 30 de setembro de 2001.

*Autor para correspondência

E-mail: jpneves@lince.hcv.ufsm.br

\begin{abstract}
RESUMO
Este trabalho teve por objetivo avaliar a eficiência de tratamentos hormonais sobre a fertilidade de vacas de corte no pós-parto com diferentes condições corporais, durante a estação de monta de outono. Setenta e três vacas pluríparas cruzadas (Hereford x Nelore) criadas extensivamente, com condição corporal entre 2 e 4, foram pesadas e distribuídas em três grupos experimentais. O grupo GSED, constituído por 25 vacas, recebeu pessário vaginal (dia 0) contendo $250 \mathrm{mg}$ de acetato de medroxiprogesterona e $500 \mathrm{mg}$ de somatotropina bovina recombinante (bST-r). Na retirada dos pessários (dia 7), as vacas receberam $0,5 \mathrm{mg}$ de cipionato de estradiol e procedeu-se o desmame temporário dos bezerros por 72 horas. No grupo SED, 25 vacas receberam tratamento semelhante ao grupo GSED, porém não receberam bST-r. No grupocontrole, as 23 vacas somente foram separadas dos seus bezerros por $72 \mathrm{~h}$. Quando da retirada dos pessários as vacas foram colocadas com touros por 30 dias. Os animais foram pesados e avaliados quanto à condição corporal no início do experimento e na retirada dos touros (dia 37). Foi constatada perda média de peso de $0,648 \mathrm{~kg} / \mathrm{dia}$ e os percentuais de estro foram de $26,1 \%, 33,3 \%$ e $56,5 \%$, respectivamente, para os grupos controle, SED e GSED. O diagnóstico de gestação, realizado pela palpação retal 60 dias após a retirada dos touros, indicou percentuais de prenhez de 13,0\%, 8,3\% e 21,7\%, respectivamente, para os grupos controle, SED e GSED $(\mathrm{P}=0,16)$, demonstrando que os programas hormonais adotados não foram eficientes no incremento das taxas de prenhez de vacas que perdiam peso entre 50 e 70 dias após o parto.
\end{abstract}

Palavras-chave: Vacas de corte, taxa de prenhez, indução de cio, somatotrofina bovina

\begin{abstract}
This study aimed to assess the efficiency of hormonal treatment in the postpartum fertility of nursing beef cows. Seventy-three cows (Hereford $x$ Nellore) raised extensively, with body condition ranging from 2 to 4 were randomly allotted in three groups. The GSED group, with 25 cows, received an intravaginal device (ID) of 250mg of medroxiprogesteron acetate and 500mg of recombinant bovine somatotropin (bST-r; day 0). After the ID withdrawal (day 7), the cows received $0.5 \mathrm{mg}$ of estradiol cipionate; their calves were temporarily weaned for 72 hours. In the SED group, 25 cows received a similar treatment, however, somatotropin was not used. In the control group, 23 cows were temporarily separated from their calves for 72 hours and did not receive any hormonal treatment. The cows were put together with
\end{abstract}


the bulls for 30 days just after ID withdrawal. The cows were weighted and assessed for body condition at day 0 and just after bulls withdrawal. The cows lost weight in an average of $0.648 \mathrm{~kg} /$ day, and $56.5 \%$, $33.3 \%$, and $26.1 \%$ of estrous rates were observed, respectively for the GSED, SED and control groups (GSED vs. SED, $P=0.0001$; GSED vs. control, $P=0.0007$; $S E D$ vs. control, $P=0.53$ ). The pregnancy rates were $21.7 \%, 8.3 \%$, and $13.0 \%$, respectively for the GSED, SED and control groups $(P=0.16)$. The results showed that the hormonal program associated with temporary 72-hour calf removal did not increase conception rate of cows that loosed weight from 50 to 70 days postpartum.

Keywords: Beef cow, pregnancy rate, estrous induction, bovine somatotrofin

\section{INTRODUÇÃO}

Diversas pesquisas têm sido realizadas visando propor alternativas que sejam eficazes na retomada da ciclicidade reprodutiva de vacas de corte no período pós-parto. Uma alternativa está na indução da ovulação de fêmeas que se encontram no período de anestro pós-parto, pela utilização de tratamentos hormonais. Embora os mecanismos hormonais que inibem o reinício da atividade cíclica fisiológica e atrasam a recuperação da ciclicidade ovariana após o parto ainda não estejam totalmente compreendidos, algumas evidências indicam que a supressão da liberação do hormônio liberador de gonadotrofinas $(\mathrm{GnRH})$ e a conseqüente inibição dos pulsos do hormônio luteinizante (LH) sejam os fatores endócrinos que conduzem ao maior período de anestro pós-parto (Gregg et al., 1986; Williams \& Griffith, 1995). Murphy et al. (1990) detectaram o primeiro folículo dominante em média 10,2 dias após o parto em vacas com razoável condição corporal. Esse folículo já possui receptores para LH nas células da granulosa, além de concentrações de progesterona, estradiol e testosterona no líquido folicular consideradas fisiológicas, o que sugere semelhança com folículos dominantes desenvolvidos durante a fase lútea do ciclo estral (Braden et al., 1986). Em vacas não submetidas a estresse nutricional, o período de anestro pós-parto ocorre mais em função de falhas da ovulação dos primeiros folículos dominantes do que de atraso ou ausência de seu desenvolvimento (Roche et al., 1992; Stagg et al., 1994). Com isso, alguns tratamentos adotados em vacas de corte durante o período pós-parto com progestágenos, associados a substâncias luteolíticas e gonadotróficas, têm sido eficientes na indução e sincronização do ciclo estral (Mulvehill \& Sreenan, 1977; Pratt et al., 1982; Roche et al., 1992; Bo et al., 1996).

Além dos hormônios citados, existem elementos que podem ser adicionados com sucesso aos programas hormonais de indução e sincronização do ciclo estral na espécie bovina, como por exemplo a somatotrofina bovina recombinante (bST-r). Essa substância, quando liberada na circulação sangüínea, estimula a produção e a secreção de IGF-1, porém suas relações com a secreção de gonadotrofinas, com o desenvolvimento folicular, com a função luteal e com a manifestação de estro ainda permanecem em dúvidas (Santos \& Amstalden, 1998). Parece estar bem estabelecido o incremento da resposta aos tratamentos hormonais nos casos onde os bezerros são separados das mães no momento da retirada dos implantes de progestágenos (Odde, 1989).

Este trabalho teve por objetivo averiguar a eficiência do uso da bST-r em sistemas de indução hormonal baseado em progesterona/estrógeno, associados ao desmame temporário, sobre a indução do estro e taxa de prenhez de vacas de corte no período pós-parto.

\section{MATERIAL E MÉTODOS}

O trabalho foi desenvolvido na Fazenda Santa Virgínia, localizada no município de Quaraí (RS), durante os meses de junho e julho de 1998. Foram utilizadas 73 vacas pluríparas cruzadas (Hereford x Nelore), criadas em campo nativo, com condição corporal (CC) variando de 2 a 4 (1=caquética e 5=obesa) entre 50 e 70 dias pós-parto. Os animais foram submetidos a exame ginecológico para avaliação da saúde genital, pesados e posteriormente distribuídos em três grupos experimentais (GSED, SED e controle), buscando-se uma distribuição uniforme com relação à condição corporal nos grupos. No momento da 
primeira pesagem (dia 0) mediram-se a altura, o diâmetro torácico e o comprimento para que fosse calculada a área de superfície corporal e o índice corporal (IC). Para obtenção do IC aplicou-se a fórmula:

$$
I C=\frac{P}{4 \times \pi \times(1 / 8 \times A \times C \times D T)^{2 / 3}},
$$

em que:

$$
\begin{aligned}
& \mathrm{P}=\text { peso vivo; } \\
& \mathrm{A}=\text { altura; } \\
& \mathrm{C}=\text { comprimento cabeça-anca; } \\
& \mathrm{DT} \text { = diâmetro torácico. }
\end{aligned}
$$

O grupo GSED, constituído por 25 vacas, recebeu no primeiro dia do experimento (dia 0) implante vaginal contendo $250 \mathrm{mg}$ de acetato de medroxiprogesterona (SINCROBOVI, EMBRAPA/CPPSUL-Bagé, RS) e 500mg de somatotrofina bovina recombinante (Boostin, Coopers Brasil Ltda. - Cotia, SP) (bST-r) por via subcutânea. No dia de retirada do pessário intravaginal (dia 7) foi administrado 0,5mg de cipionato de estradiol (ECP, Rhodia-Merieux Veterinária Ltda.,Paulínia-SP) por via intramuscular e feita a separação dos bezerros por 72h. No grupo SED, 25 vacas receberam tratamento semelhante ao anterior, porém não receberam bST-r. O grupo-controle, formado por 23 vacas, não recebeu tratamento hormonal, adotandose somente a separação dos bezerros por $72 \mathrm{~h}$. No momento da retirada dos implantes todas receberam tinta-cola (Kerr \& McCaughey, 1984), que consiste na pintura da inserção da cauda entre a primeira e a sexta vértebras caudais, utilizada como critério de avaliação de ocorrência de estro. As vacas foram colocadas em piquetes com touros (dia 7), na proporção de um touro para cada 10 vacas, onde permaneceram por 30 dias. Sete dias após a introdução nos piquetes a resposta (estro) aos tratamentos foi avaliada por observação da tinta-cola. Quando da retirada dos touros (dia 37), as vacas foram novamente pesadas. O diagnóstico de gestação foi realizado por palpação retal 60 dias após a retirada dos touros.

Usou-se o teste de qui-quadrado para analisar a resposta quanto ao estro e à prenhez. A correlação entre condição corporal e índice corporal foi analisada por meio de regressão linear (Proc GLM) utilizando-se o programa estatístico SAS (1988).

\section{RESULTADOS E DISCUSSÃO}

O percentual de vacas que manifestaram estro após os tratamentos encontra-se na Tab. 1. Os resultados sugerem que a resposta estral pode ter sido estimulada pela ação da bST-r, a qual já foi associada ao aumento nos níveis sangüíneos de estradiol-17 (Andrade et al., 1996), resultante da ação da bST-r sobre a foliculogênese. Neste estudo a bST-r foi administrada sete dias antes do programa de indução de cios. Considerando-se que o produto utilizado apresenta liberação lenta e a dose administrada mantém os níveis séricos de IGF por 14 dias, ele ainda estaria atuando no momento da retirada dos pessários intravaginais. Entre os grupos SED e controle não foi verificada diferença quanto ao percentual de resposta estral $(\mathrm{P}=0,53)$, sugerindo que o uso do estradiol e dos pessários de gestágenos associado à separação temporária não foi eficiente em incrementar a resposta estral. Isso pode ter ocorrido em função da pequena dose de estradiol utilizada $(0,5 \mathrm{mg} / \mathrm{animal})$, já que a quantidade mínima de estradiol a ser administrada deveria ser de $1 \mathrm{mg}$ em vacas no período pós-parto, conforme Lammoglia et al. (1998).

Não foram observadas diferenças entre os grupos quanto à taxa de prenhez $(\mathrm{P}=0,16)$. Os percentuais de vacas que resultaram prenhes em relação às que manifestaram estro, dentro de cada grupo, foram de $13,1 \%$ no controle, $25,0 \%$ no SED e $34,8 \%$ no GSED. O programa adotado mostrou-se eficiente em induzir a resposta ao estro, porém não aumentou os índices de prenhez e, conseqüentemente, a eficiência 
reprodutiva dos animais. Essas diferenças sugerem a presença de sinais de estro estimulada pelos tratamentos sem que ocorra ovulação ou indução de ciclicidade, aspectos já descritos por Nelsen et al., (1985), Rutter \& Randel (1986) e Callejas et al. (1995). O baixo percentual de prenhez em relação ao de estro pode ser conseqüência de falhas das fêmeas em ovularem, em manterem a ciclicidade, em conceberem ou em manterem a gestação.

Tabela 1. Percentual de estro e prenhez, média e erro-padrão da média de peso, da condição corporal e de índice corporal nos grupos GSED (somatotrofina, progestágenos, estradiol e separação dos bezerros), SED (progestágeno, estradiol e separação dos bezerros) e controle (separação dos bezerros) em vacas Hereford x Nelore.

\begin{tabular}{lccc} 
& \multicolumn{3}{c}{ Grupo } \\
\cline { 2 - 4 } & GSED & SED & Controle \\
\hline Estro $(\%)$ & $56,5 \mathrm{a}$ & $33,3 \mathrm{~b}$ & $26,1 \mathrm{~b}$ \\
Prenhez $(\%)$ & $21,7 \mathrm{a}$ & $8,3 \mathrm{a}$ & $13,0 \mathrm{a}$ \\
Peso inicial & $380,6 \pm 18,8$ & $363,2 \pm 20,4$ & $392,2 \pm 14,5$ \\
Peso final & $369,1 \pm 7,7$ & $353,9 \pm 11,1$ & $360,4 \pm 12,8$ \\
Condição corporal inicial & $3,1 \pm 0,1$ & $2,9 \pm 0,1$ & $3,1 \pm 0,2$ \\
Condição corporal final & $2,9 \pm 0,1$ & $2,7 \pm 0,1$ & $2,8 \pm 0,1$ \\
Índice corporal inicial & $63,8 \pm 1,1$ & $61,4 \pm 1,4$ & $63,3 \pm 1,7$ \\
Índice corporal final & $58,8 \pm 1,0$ & $57,5 \pm 1,1$ & $58,2 \pm 1,3$ \\
\hline
\end{tabular}

Valores seguidos por letras iguais na linha não diferem entre si ( $\mathrm{P}>0,05)$.

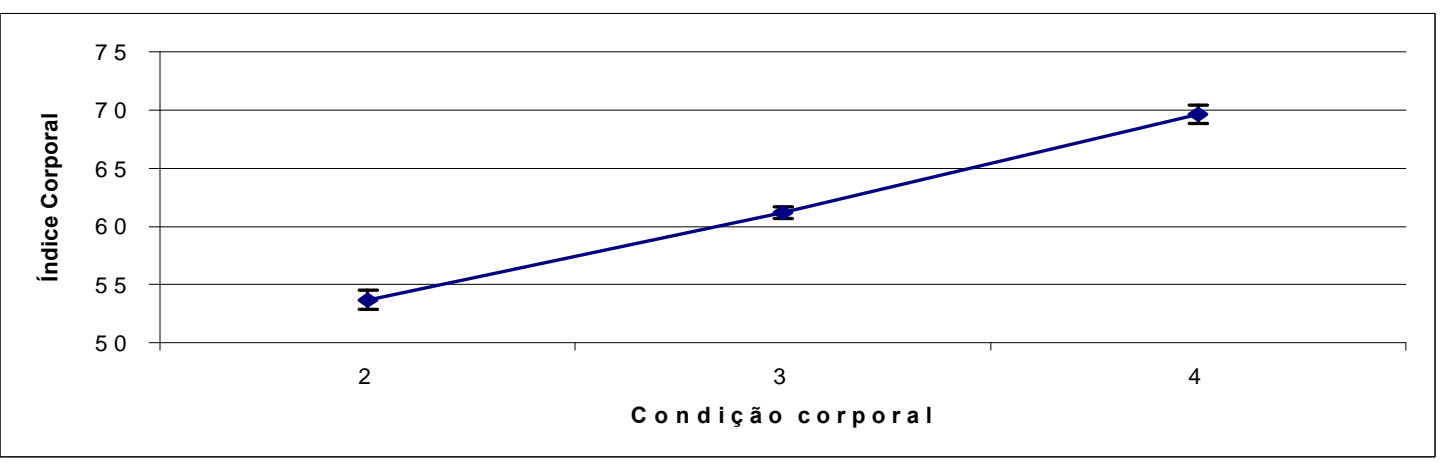

Figura 1. Correlação e erro-padrão da média entre condição corporal e índice corporal em vacas Hereford $\mathrm{x}$ Nelore.

Não foi observada associação entre prenhez e CC ou IC dos animais provavelmente em função do pequeno número de vacas de diferentes $\mathrm{CC}$ em cada grupo. Observou-se correlação linear $(\mathrm{P}=0,0001)$ entre CC e IC. O IC médio foi de 53,6, 61,1 e 69,6 para vacas com CC 2, 3 e 4, respectivamente (Fig. 1). Considerando que a avaliação da condição corporal varia em função da raça, do tamanho do animal e do avaliador, considerou-se importante adotar uma medida objetiva de avaliação do estado nutricional dos animais. Os resultados observados indicam que, por considerar a superfície corporal e o peso do animal e, portanto, caracterizar fisicamente o indivíduo avaliado, o IC pode constituir-se em um método objetivo de avaliação, o qual poderá substituir a avaliação subjetiva da CC. 
Conclui-se que o uso do bST-r associado ao sistema de indução hormonal baseado em progesterona, estrógeno e desmame temporário por $72 \mathrm{~h}$ aumenta os índices de manifestação de estro, mas não incrementa as taxas de prenhez de vacas de corte que estão perdendo peso entre 50 e 70 dias após o parto.

\section{REFERÊNCIAS BIBLIOGRÁFICAS}

ANDRADE, L.P., RHIND, S.M., WRIGHT, I.A. et al. Effects of bovine sometotropin (bST) on ovarian function in post-partum beef cows. Reprod. Fert. Develop., v.8, p.951-960, 1996.

BO, G.A., ADAMS, G.P., PIERSON, R.A. et al. Effect of progestogen plus estradiol-17 $\beta$ treatment on superovulatory response in beef cattle. Theriogenology, v.45, p.897-910, 1996.

BRADEN, T.D., MANNS, J.G., CERMAK, D.L. et al. Follicular development following parturition and during the estrous cycle in beef cows. Theriogenology, v.25, p.833-843, 1986.

CALlEJAS, C., ALBERIO, R., DORAY, J. et al. Postpartum sexual activity in beef cows after temporary calf removal with or without oestradiol benzoate. Rev. Méd. Vet., v.75, p.363-367, 1995.

GREGG, D.W., MOSS, G.E., HUDGENS, R.E. et al. Endogenous opioid modulation of luteinizing hormone and prolactin secretion in postpartum ewes and cows. J. Anim. Sci., v.63, p.838-847, 1986.

KERR, O.M., McCAUGHEY, W.J. Tail painting technique as an aid to oestrus detection in cattle. Vet. Res., v.114, p.605-607, 1984.

LAMMOGLIA, M.A., SHORT, R.E., BELLOWS, S.E. et al. Induced and syncronization estrus in cattle: Dose titration of estradiol benzoate in peripubertal heifers and postpartum cows after treatment with an intravaginal progesterone-releasing insert and prostaglandin F2 . J. Anim. Sci., v.76, p.1662-1670, 1998.

MULVEHILL, P., SREENAN, J. Improvement of fertility in postpartum beef cows by treatment with PMSG and progestogen. J. Reprod. Fert., v.50, p.323-325, 1977.

MURPHY, M.G., BOLAND, M.P., ROCHE, J.F. Pattern of follicular growth and resumption of ovarian activity in post-partum beef suckler cows. J. Reprod. Fert., v.90, p.523-533, 1990.

NELSEN, T.C., SHORT, R.E., PHELPS, D.A. et al. Nonpuberal estrus and mature cow influences on growth and puberty in heifers. J. Anim. Sci., v.61, p.470, 1985.

ODDE,K.G. A review of syncronization of estrus in postpartum cattle. J. Anim. Sci., v. 68, p. 817-830, 1989.

PRATT, B.R., BERARDINELLI, J.G., STEVENS, L.P. Induced corpora lutea in the postpartum beef cow. I. Comparison of gonadotropin releasing hormone and human chorionic gonadotropin and effects of progesterone and estrogen. J. Anim. Sci., v.54, p.822-830, 1982.

ROCHE, J.F., CROWE, M.A., BOLAND, M.P. Postpartum anoestrus in dairy and beef cows. Anim. Reprod. Sci., v.28, p.371-378, 1992.

RUTTER, L.M., RANDEL, R.D. Nonpuberal estrus in beef heifers. J. Anim. Sci., v.63, p.1049-1053, 1986.

SAS. Statistical analysis sistem. 3.ed. Cary: SAS Institute INC, 1988. 1028 p.

SANTOS, J.E.P., AMSTALDEN, M. Effects of nutrition on bovine reproduction. Arq. Fac. Vet. UFRGS, v.26, p. 1989, 1998.

STAGG, K., DISKIN, M.G., SREENAN, J. M. et al. Follicular development in long-term anoestrous suckled beef cows fed two levels of energy postpartum. Anim. Reprod. Sci., v.38, p.49-61, 1994.

WILLIAMS, G.L., GRIFFITH, M.G. Sensory and behavioural control of gonadotrophin secretion during sucklingmediated anovulation in cows. J. Reprod. Fert., v.49, p.464-475, 1995. 